\title{
Micro-computed tomography assessment of human femoral trabecular bone for two disease groups (fragility fracture and coxarthrosis): Age and gender related effects on the microstructure
}

\author{
Ana Catarina Vale ${ }^{1,2}$, Manuel F. C. Pereira ${ }^{3}$, António Maurício ${ }^{3}$, Bruno Vidal ${ }^{2}$, Ana Rodrigues ${ }^{2,4}$, \\ Joana Caetano-Lopes ${ }^{2}$, Ara Nazarian ${ }^{5}$, João E. Fonseca, ${ }^{2,4}$, Helena Canhão ${ }^{2,4}$, Maria Fátima Vaz ${ }^{1,6^{*}}$
}

\footnotetext{
${ }^{1}$ Institute of Materials and Surface Science and Engineering, Lisbon, Portugal

${ }^{2}$ Rheumatology Research Unit, Instituto de Medicina Molecular, Faculdade de Medicina de Lisboa, Lisbon, Portugal

${ }^{3}$ Center of Petrology and Geochemistry, Instituto Superior Técnico, Universidade Técnica de Lisboa, Lisbon, Portugal

${ }^{4}$ Rheumatology and Metabolic Bone Diseases Department, Hospital de Santa Maria, Lisbon, Portugal

${ }^{5}$ Orthopedic Biomechanics Laboratory, Beth Israel Deaconess Medical Center, Harvard Medical School, Boston, USA

${ }^{6}$ Mechanical Engineering Department, Instituto Superior Técnico, Universidade Técnica de Lisboa, Lisbon, Portugal

Email: ${ }^{*}$ fatima.vaz@,ist.utl.pt
}

Received 16 November 2012; revised 15 December 2012; accepted 22 December 2012

\begin{abstract}
The aim of this study was to identify three-dimensional microstructural changes of trabecular bone with age and gender, using micro-computed tomography. Human trabecular bone from two disease groups, osteoporosis and osteoarthritis was analyzed. A prior analysis of the effects of some procedure variables on the micro-CT results was performed. Preliminary micro-CT scans were performed with three voxel resolutions and two acquisition conditions. On the reconstruction step, the image segmentation was performed with three different threshold values. Samples were collected from patients, with coxarthrosis (osteoarthritis) or fragility fracture (osteoporosis). The specimens of the coxarthrosis group include twenty females and fifteen males, while the fragility fracture group was composed by twenty three females and seven males. The mean age of the population was $69 \pm$ 11 (females) and $67 \pm 10$ years (males), in the coxarthrosis group, while in the fragility fracture group was $81 \pm 6$ (females) and $78 \pm 6$ (males) years. The 30 $\mu \mathrm{m}$ voxel size provided lower percentage difference for the microarchitecture parameters. Acquisition conditions with $160 \mu \mathrm{A}$ and $60 \mathrm{kV}$ permit the evaluation of all the volume's sample, with low average values of the coefficients of variation of the microstructural parameters. No statistically significant differences were found between the two diseases groups, neither between genders. However, with aging, there
\end{abstract}

${ }^{*}$ Corresponding author. is a decrease of bone volume fraction, trabecular number and fractal dimension, and an increase of structural model index and trabecular separation, for both disease groups and genders. The parameters bone specific surface, trabecular thickness and degree of anisotropy have different behaviors with age, depending on the type of disease. While in coxarthrosis patients, trabecular thickness increases with age, in the fragility fracture group, there is a decrease of trabecular thickness with increasing age. Our findings indicate that disease, age and gender do not provide significant differences in trabecular microstructure. With aging, some parameters exhibit different trends which are possibly related to different mechanisms for different diseases.

Keywords: Trabecular Bone; Micro-Computed Tomography; Coxarthrosis; Fragility Fracture; Age; Gender

\section{INTRODUCTION}

As for other materials, the mechanical properties of bone depend on its structural characteristics. Trabecular bone is formed by an interconnected network of rods and plates and can be found at the epiphysis of long bones and in the vertebral body. Almost all fragility fractures occur at regions with trabecular bone, for which the trabecular microarchitecture was affected by a disease mechanism. In this sense, the bone structural characterization, in particular of trabecular bone, is fundamental to assess the risk of fracture and to help in the prevention of bone 
failure.

Osteoarthritis (OA) and osteoporosis (OP) are pathologies that affect the quality of life of patients. Osteoporosis occurs due to the discrepancy between bone formation and bone resorption, which may lead to bone loss, associated to an increase on the risk of bone failure. In this sense, the trabecular microarchitecture is an important determinant of osteoporosis. Osteoarthritis is a chronic inflammatory disease which mainly affects the cartilage of the joint compromising the bone properties, whose influence on the microstructure of bone is not very well known.

Micro-computed tomography (micro-CT) has become an important tool to the visualization and quantification of the three-dimensional (3D) structure of bone [1-15] instead of inferring the properties from 2D measurements, which is the case of histomorphometric evaluation. Nevertheless, there are very good correlations between histomorphometric and microtomographic analysis $[16,17]$. Micro-CT has been used to evaluate differences of trabecular microstructure, e.g. between several age groups [18-21] at different anatomical locations [18,22,23]. As osteoporosis generates morphological alterations of the bone structure, an accurate study of trabecular bone microarchitecture can be performed by micro-CT to study the origin of the disease, as well as, to evaluate its evolution [24-34]. Additionally, some studies have been focused on trabecular bone samples from animals or humans with disorders associated to osteoarthritis [30,3438]. Micro-CT is also used to assess microarchitectural changes during mechanical testing [39-42].

Micro-CT is widely used to evaluate the microstructure of trabecular bone and is regarded to be a gold standard technique. Depicted the number of papers on this subject, some aspects may be missing. Although some works refer to the comparison between diseases as osteoporosis and osteoarthritis [30,35], while others mention the age and gender effect on the structure $[20,21]$ a survey of the literature did not allow to find any combination of the age, gender and disease.

Although, there are studies on the micro-computed tomography applied to trabecular bone, which deal with the impact of the procedure variables on the accuracy of the measurements, each case is a different case with its own particular characteristics depending on the apparatus properties, process variables and sample properties $[2,43$, 44]. Prior to the application of the micro-CT technique to a large number of samples, a study on the effects of some procedure variables is advantageous. In this work, an optimization of the micro-CT parameters with emphasis to the voxel size, voltage, intensity and threshold values was performed.

The aim of this study was to identify three-dimensional (3D) microarchitectural changes of trabecular bone simultaneously with age, gender, and two different pathologies, coxarthrosis (CA) and fragility fracture (F) using micro-computed tomography (micro-CT). We hypothesized that, in the diseased samples, there would be age-related changes in the trabecular bone microarchitecture, in similitude to age effects in healthy bone.

\section{EXPERIMENTAL PROCEDURE}

\subsection{Materials}

Femoral epiphyses were collected from patients submitted to hip replacement surgery in the Orthopedic Department of Hospital de Santa Maria, Lisbon, Portugal. Two pathologies were evaluated, namely coxarthrosis which is a particular form of osteoarthritis, and fragility fractures, which probably occurred due to osteoporosis. Trabecular bone cylinders were drilled to extract cylinder-shaped samples of $5 \mathrm{~mm}$ in diameter and approximately $15-20 \mathrm{~mm}$ in length. This study was approved by local Ethics Committee and followed the International Guidelines stated by Declaration of Helsinki (Seoul, 2008). Patient's agreement to these experiments was obtained by written informed consent.

Bone cylinders were prepared following a procedure which included five steps: fixation, dehydration, clearing, impregnation and inclusion [45]. During the fixation phase, the sample is placed in alcohol $70 \%$ for a minimum of 72 hours at $5^{\circ} \mathrm{C}$, after which they were dehydrated in ethanol $96 \%$ to $100 \%$, over a period of 24 hours, at $5^{\circ} \mathrm{C}$. On the third step, samples were cleared in order to replace the alcohol by an intermediate solution of xylene for 24 hours at $5^{\circ} \mathrm{C}$. Then, the specimens were embedded in methylmethacrylate (MMA) for a minimum of 72 hours at $-20^{\circ} \mathrm{C}$, after which they were included in the polymer at a constant temperature between $5^{\circ} \mathrm{C}$ and $10^{\circ} \mathrm{C}$, to polymerize [46].

Initially, three trabecular bone samples from patients with CA were studied, which belong to two male patients with mean age of $64 \pm 7$ years, and one female with 61 years to establish imaging parameters to be used in this study. Then, a total of sixty-five samples were analyzed and two bone diseases were compared, using thirty-five coxarthrosis specimens (20 females with mean age of 69 years, 5 males with mean age of 67 years) and thirty fragility fracture specimens ( 23 females with mean age of 81 years, 7 males with mean age of 78 years).

\subsection{Procedure Variables in the Micro-CT Evaluation}

The goal of the first part of the work was to establish an optimized methodology to measure 3D trabecular bone structural characteristics, in order to apply it to a wider sample population. For this purpose a set of three sam- 
ples was used. Measurements were determined three times for each of the samples, under the same testing conditions.

Micro-CT analysis involves three main steps: acquisition, reconstruction and image analysis [3]. In the acquisition stage, the variables to be chosen are the X-ray tube potential, voxel size, and location of the volume of interest [3]. On the reconstruction phase, the binarization (or segmentation) is carried out, separating bone from no bone regions. Despite the number of methods found on the literature, no completely reliable method of bone segmentation has been established and an optimal thresholding protocol should be proposed for each case studied. In general, for bone samples, local threshold are preferred, especially for comparison studies with histomorphometric evaluation [16]. On the third phase, the reconstructed image data can be interpreted with a $3 \mathrm{D}$ analysis software that enables the quantification of the bone microstructure parameters. Although several parameters may be used to characterize the 3D structure of trabecular bone, the most widely used are $[3,47,48]$ : Percent bone volume or Bone volume fraction ( $\mathrm{BV} / \mathrm{TV}, \%)$, Bone specific surface (BS/BV $\mathrm{mm}^{-1}$ ), Structure model index (SMI, none), Trabecular thickness (Tb.Th, mm), Trabecular number (Tb.N, $\mathrm{mm}^{-1}$ ), Trabecular separation (Tb.Sp, $\mathrm{mm}$ ), Fractal dimension (FD, none), and Degree of anisotropy (DA, none).

The micro-CT study was performed on a SkyScan 1172 device (SkyScan, Kontich, Belgium) [49]. The apparatus is controlled by a computer programmed with the SkyScan software package, which is based on Feldkamp algorithm [1]. Figure 1 shows an example of the shadow image (or projection image), the reconstructed slice, the region of interest (ROI), the binarized region, and the $3 \mathrm{D}$ rendering (i.e., manipulation in a virtual space) of a human trabecular bone sample.

The acquisition protocol involves several scan parameters, some of which were changed to verify their influence in the results. The X-ray projection images were collected under two conditions of X-ray voltage and Xray current denoted respectively by $A$ ) and $B$ ), where A) corresponds to $160 \mu \mathrm{A}$ and $60 \mathrm{kV}$ and $\mathrm{B}$ ) to $100 \mu \mathrm{A}$ and $100 \mathrm{kV}$. Images were acquired over an angular range of $180^{\circ}$ with an angular step of $0.45^{\circ}$, with scanning times around $30 \mathrm{~min}$. The tested values of the image pixel size were 10,15 and $30 \mu \mathrm{m}$. During the micro-CT scan, each

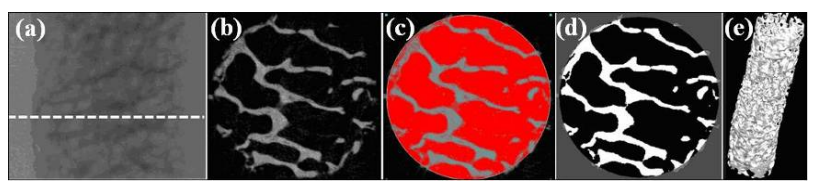

Figure 1. (a) Projection image; (b) Reconstructed slice; (c) Region of interest (ROI); (d) Binarized section; and (e) 3D rendering. sample was entirely contained in the field of view (FOV) and a stack of 1000 , or more, cross-section images was obtained with a slice to slice increment of $10 \mu \mathrm{m}$. The projection images were stored in TIFF file format, as 16-bit shadow images, in a size of matrix around $640 \times$ 512 pixels.

The size and position of the volumes of interest were chosen by the operator to obtain the maximum possible volume. Samples were only repositioned between each image acquisition and the analysis was conducted by the same operator.

Following scanning, the projection images were reconstructed, in about 500 slices along the $\mathrm{ZZ}$ axis, by using the cone-beam reconstruction software NRecon (SkyScan, Kontich, Belgium) [49]. The segmentation method applied is based on the local minimum of the grayscale bimodal histogram function of the stack of slices images corresponding to the volume of interest. Three different threshold values on the histogram function were tested which correspond to 1) the grey value of the local minimum $\left.\left(\mathrm{g}_{\min }\right) ; 2\right)$ the minimum value minus 5 units of the grey level scale $\left(g_{\min }-5\right)$; and 3$)$ the minimum value plus 5 units $\left(g_{\text {min }}+5\right)$. Each sample has a unique $g_{\min }$ and therefore the threshold values were different for distinct samples. An example is given in Figure 2.

On the third step, the reconstructed slice images were processed, quantified and interpreted by means of 3D image analysis software (CTAn and ANT software, SkyScan, Kontich, Belgium). This 3D rendering enabled the determination of the previously mentioned structural parameters for the analysis of trabecular structure.

The reproducibility of measurements of the morphological parameters was assessed by determining these parameters, making three scans for each sample, under the same acquisition conditions, and calculating the coefficients of variation, $\mathrm{CV}(\%)$ given by Eq. 1. To compare the results of the two scanning conditions (p.A, p.B), the percent difference, $\Delta \mathrm{Diff}(\%)$, was also evaluated with the Eq. 2.

$$
\begin{array}{r}
\operatorname{CV}(\%)=\text { Standard Deviation } / \text { Mean } \\
\Delta \operatorname{Diff}(\%)=200 \times \mid(\text { p.A }- \text { p.B }) \mid /(\text { p.A }+ \text { p.B })
\end{array}
$$

A comparison between the two conditions A) and B) for the three voxel sizes $(10,15$ and $30 \mu \mathrm{m})$, is evaluated by the $\Delta$ Diff $(\%)$ values, which are illustrated on Figure 3.

In general, the percentage difference for the microarchitectural parameters is lower for the results obtained with $30 \mu \mathrm{m}$ voxel size, with the exceptions of BV/TV and DA. Some works report that the scanning of large specimens may require the use of special resolutions correspondent to voxel sizes greater than $100 \mu \mathrm{m}$ [2]. 


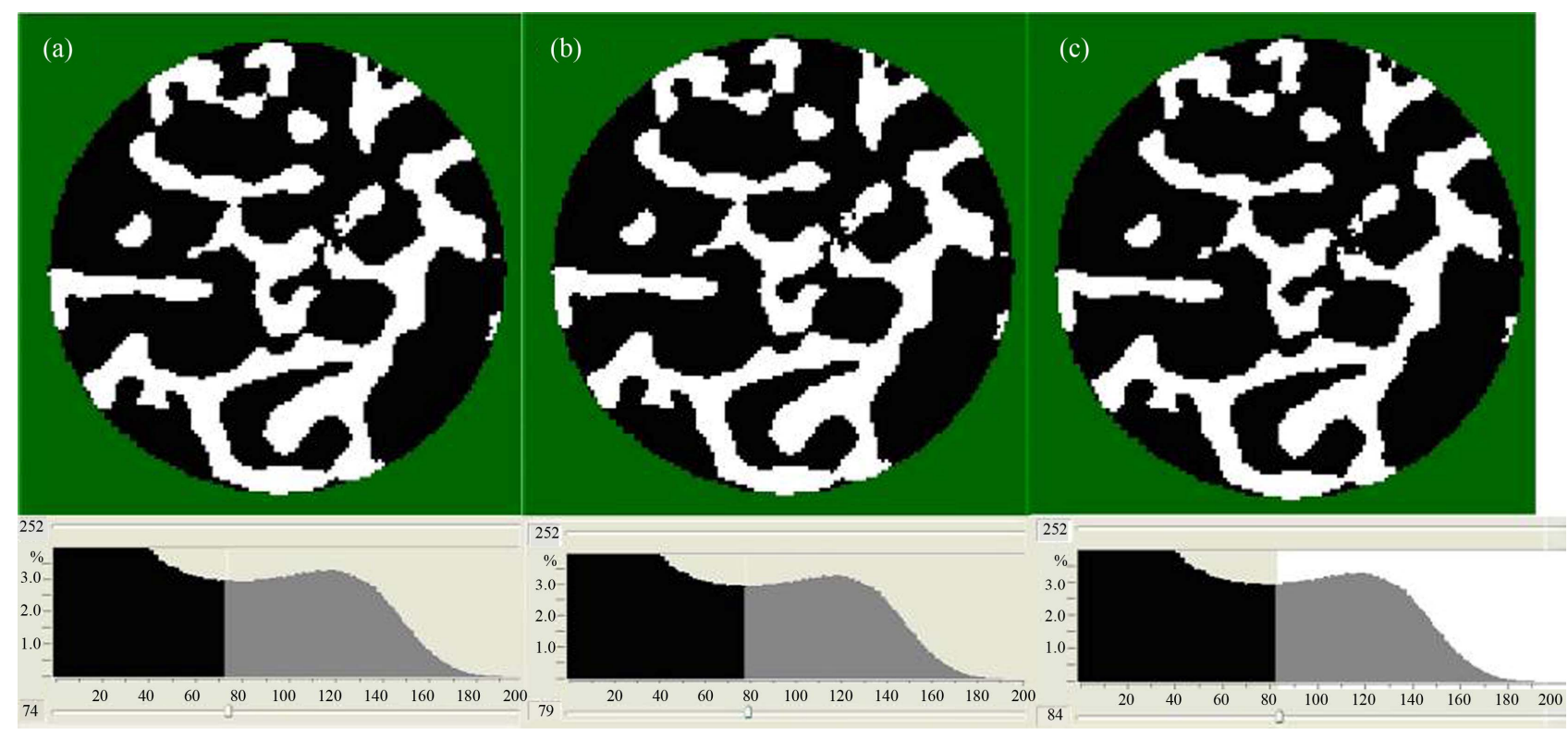

Figure 2. Effect of the grayscale histogram thresholding value on the binarized ROI: (a) $g_{\min }-5$; (b) $g_{\min }$; and (c) $g_{\min }+5$, where $\mathrm{g}_{\min }$ is the grey level correspondent to the minimum (A scan conditions: $160 \mu \mathrm{A}$ and $60 \mathrm{kV}, 30 \mu \mathrm{m}$ pixel size).

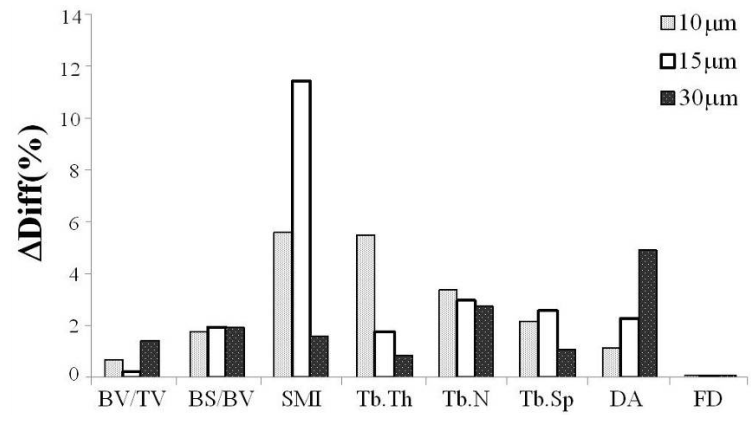

Figure 3. Percent difference, $\Delta \operatorname{Diff}(\%)$, between the mean values from the two acquisition conditions, $\mathrm{A}$ and $\mathrm{B}$, for three voxel sizes $(10,15$ and $30 \mu \mathrm{m})$.

However, for voxel sizes larger than $100 \mu \mathrm{m}$, the microarchitectural parameters are strongly dependent on the voxel size [2]. As, when the voxel size decreases, the resolution increases, it is convenient to choose a voxel size lower than $100 \mu \mathrm{m}$, which allows scanning the entire sample's volume. The voxel size of $30 \mu \mathrm{m}$ was the ideal value for our samples.

Table 1 presents the microarchitectural parameters, for the three samples, determined under scan conditions $\mathrm{A}$ and $\mathrm{B}$, with a voxel size of $30 \mu \mathrm{m}$. The average values of the coefficients of variation are almost the same either for settings A or B conditions.

As there is not much influence of the acquisition voltage and current on the results, A conditions were chosen to evaluate the rest of the samples, as it allows to evaluate all the volume's sample and reduces the artifacts. This is in accordance with authors that recommend the use of micro-CT works at the medium range of X-ray energy (50 to $90 \mathrm{kV}$ ) [3].

We determined the threshold influence on a set of three samples, using three values of the global grayscale histogram, $g_{\min }-5, g_{\min }$ and $g_{\min }+5$. Table 2 quantifies the threshold effect on the binarized BVI for one sample, where the microarchitectural parameters determined under different values of the grayscale histogram threshold, are shown. The average of the three results is very similar to the results obtained with the $\mathrm{g}_{\text {min }}$ value. The CVs associated with the measurements are in the interval of $0.5 \%$ to $8 \%$. The higher $\mathrm{CV}$, around $8 \%$, was also obtained for $\mathrm{BV} / \mathrm{TV}$, while the coefficient of variation for $\mathrm{BS} / \mathrm{BV}$ and $\mathrm{Tb}$. Th varied between $6 \%$ to $7 \%$. For SMI, the $\mathrm{CV}$ value is $4.36 \%$ and the coefficients for $\mathrm{BS} / \mathrm{TV}$, Tb.N, Tb.Sp, DA and FD are equal or lower than $2 \%$. Our values are in the range of a similar work performed by Beaupied et al. [44]. We decided to use values of $\mathrm{g}_{\text {min }}$ which provides results similar to the average of the results obtained with these three threshold values.

\subsection{Micro-CT Imaging}

Taking into account the preliminary results obtained in the study, the optimal parameters for scanning and reconstruction of trabecular bone were chosen as: acquisition conditions of voltage equal to $60 \mathrm{kV}$, intensity of $160 \mu \mathrm{A}, 30 \mu \mathrm{m}$ of voxel size and the threshold value adjusted at the minimum of the global grayscale histogram from each specimen evaluated.

\subsection{Statistical Analysis}

First, a Shapiro Wilk test was conducted to evaluate the 
Table 1. Effect of the scan acquisition conditions A and B (A: $160 \mu \mathrm{A} / 60 \mathrm{kV}, \mathrm{B}: 100 \mu \mathrm{A} / 100 \mathrm{kV})$ on the microarchitectural parameters for three different samples (Mean \pm SD values and $C V$ ), for a voxel size of $30 \mu \mathrm{m}$.

\begin{tabular}{|c|c|c|c|c|c|c|c|}
\hline \multirow{2}{*}{ Scan conditions } & \multirow[b]{2}{*}{ Parameter } & \multicolumn{2}{|c|}{ Sample 1} & \multicolumn{2}{|c|}{ Sample 2} & \multicolumn{2}{|c|}{ Sample 3} \\
\hline & & Mean \pm SD & CV (\%) & Mean \pm SD & CV (\%) & Mean \pm SD & CV (\%) \\
\hline \multirow{8}{*}{ A } & BV/TV(\%) & $27.12 \pm 0.05$ & 0.18 & $31.87 \pm 0.06$ & 0.19 & $25.80 \pm 0.08$ & 0.31 \\
\hline & $\mathrm{BS} / \mathrm{BV}\left(\mathrm{mm}^{-1}\right)$ & $13.60 \pm 0.04$ & 0.29 & $14.59 \pm 0.05$ & 0.34 & $18.55 \pm 0.05$ & 0.27 \\
\hline & SMI & $0.98 \pm 0.04$ & 4.08 & $1.05 \pm 0.09$ & 8.57 & $1.16 \pm 0.07$ & 6.03 \\
\hline & Tb.Th (mm) & $0.25 \pm 0.03$ & 12.00 & $0.24 \pm 0.03$ & 12.50 & $0.20 \pm 0.03$ & 15.00 \\
\hline & Tb.N $\left(\mathrm{mm}^{-1}\right)$ & $1.08 \pm 0.02$ & 1.85 & $1.30 \pm 0.03$ & 2.31 & $1.31 \pm 0.05$ & 3.82 \\
\hline & Tb.Sp (mm) & $0.64 \pm 0.02$ & 3.13 & $0.49 \pm 0.01$ & 2.04 & $0.51 \pm 0.01$ & 1.96 \\
\hline & DA & $2.04 \pm 0.01$ & 0.49 & $1.96 \pm 0.01$ & 0.51 & $2.12 \pm 0.01$ & 0.47 \\
\hline & FD & $2.25 \pm 0.00$ & 0.00 & $2.28 \pm 0.00$ & 0.00 & $2.25 \pm 0.01$ & 0.44 \\
\hline \multirow{10}{*}{ B } & average & & 2.75 & & 3.31 & & 3.53 \\
\hline & BV/TV (\%) & $27.29 \pm 0.05$ & 0.18 & $29.10 \pm 0.07$ & 0.24 & $27.22 \pm 0.05$ & 0.18 \\
\hline & $\mathrm{BS} / \mathrm{BV}\left(\mathrm{mm}^{-1}\right)$ & $13.52 \pm 0.04$ & 0.30 & $15.02 \pm 0.05$ & 0.33 & $17.30 \pm 0.06$ & 0.35 \\
\hline & SMI & $0.95 \pm 0.04$ & 4.21 & $1.17 \pm 0.06$ & 5.13 & $1.02 \pm 0.12$ & 11.76 \\
\hline & Tb.Th (mm) & $0.25 \pm 0.03$ & 12.00 & $0.24 \pm 0.03$ & 12.50 & $0.21 \pm 0.04$ & 19.05 \\
\hline & Tb.N $\left(\mathrm{mm}^{-1}\right)$ & $1.08 \pm 0.02$ & 1.85 & $1.21 \pm 0.03$ & 2.48 & $1.30 \pm 0.05$ & 3.85 \\
\hline & Tb.Sp (mm) & $0.63 \pm 0.01$ & 1.59 & $0.51 \pm 0.02$ & 3.92 & $0.50 \pm 0.02$ & 4.00 \\
\hline & DA & $2.25 \pm 0.00$ & 0.00 & $2.00 \pm 0.00$ & 0.00 & $2.18 \pm 0.01$ & 0.46 \\
\hline & FD & $2.25 \pm 0.00$ & 0.00 & $2.26 \pm 0.00$ & 0.00 & $2.27 \pm 0.01$ & 0.44 \\
\hline & average & & 2.52 & & 3.08 & & 5.01 \\
\hline
\end{tabular}

Table 2. Microarchitectural parameters determined under the same acquisition conditions (15 $\mu \mathrm{m}$ voxel size, scan acquisition condition $\mathrm{A}$ and with different values of the grayscale histogram threshold, namely, $g_{\min }-5$, $g_{\min }$ and $g_{\min }+5$, where $g_{\min }$ is the grayscale minimum (Mean, standard deviation, SD, and coefficient of variation, CV).

\begin{tabular}{ccccccc}
\hline & $\mathrm{g}_{\min }-5$ & $\mathrm{~g}_{\min }$ & $\mathrm{g}_{\min }+5$ & Mean & SD & CV (\%) \\
\hline BV/TV (\%) & 35.04 & 31.32 & 30.14 & 32.17 & 2.56 & 7.95 \\
BS/BV (mm $\left.{ }^{-1}\right)$ & 13.70 & 15.23 & 15.68 & 14.87 & 1.04 & 6.98 \\
SMI (none) & 0.88 & 0.95 & 0.95 & 0.93 & 0.04 & 4.36 \\
Tb.Th (mm) & 0.24 & 0.24 & 0.22 & 0.24 & 0.01 & 6.24 \\
Tb.N (mm $\left.{ }^{-1}\right)$ & 1.43 & 1.40 & 1.37 & 1.40 & 0.03 & 2.14 \\
Tb.Sp (mm) & 0.48 & 0.49 & 0.50 & 0.49 & 0.01 & 2.04 \\
DA (none) & 1.77 & 1.82 & 1.82 & 1.80 & 0.03 & 1.60 \\
FD (none) & 2.13 & 2.15 & 2.15 & 2.14 & 0.01 & 0.54 \\
\hline
\end{tabular}

normality of the distributions. The test indicated that all bone microarchitectural parameters had non-normal distributions. Therefore, the Mann-Whitney (Wilcoxon) test was performed to assess comparisons between female and male population, and also between the two bone diseases groups.

In addition, the univariate correlation given by the Spearman's correlation coefficient of age as a continuous variable for each bone microarchitectural parameter was tested. Finally, for each bone disease group, Spearman's correlation coefficients were obtained for correlation between each microarchitectural parameter.

Statistical analysis was performed using a statistical software SAS (version 9.2, Institute Inc., Cary, NC, USA) and differences were considered statistically significant between groups for two-side p-value lower than 0.05, and for $\mathrm{p}$-value lower than 0.0001 , it was considered that those differences were highly statistically significant.

\section{RESULTS}

Table 3 presents the global results obtained from the extended trabecular microarchitectural study, where a comparative microarchitectural evaluation between two bone diseases and both genders was made. The MannWhitney (Wilcoxon) test revealed no significant differences between disease and gender. For both diseases, specimens from male and female donors presented nearly identical BV/TV values, and for these reasons the 3D reconstruction of male and female samples did not present great microarchitectural differences. Figure $\mathbf{4}$ shows the 3D reconstruction for coxarthrosis samples and Figure 5 presents the fragility fracture samples.

In order to evaluate the effect of age on microarchitectural properties, the Spearman correlation coefficients were determined for all the samples from both groups of coxarthrosis and fragility fracture (Table 4). Negative coefficients were obtained for both $\mathrm{CA}$ and $\mathrm{F}$ groups, meaning that the bone volume fraction, trabecular number and fractal dimension decrease with increasing age.

Trabecular separation and the Structure Model Index increase with age, also for both disease groups. The parameters $\mathrm{BS} / \mathrm{BV}, \mathrm{Tb}$.Th and DA show different Spear- 
Table 3. Descriptive statistics of the micro-CT measurements for the two bone disease groups, CA (coxarthrosis) and F (osteoporosis or fragility fracture).

\begin{tabular}{ccccccccc}
\hline & \multicolumn{9}{c}{ CA } & \multicolumn{3}{c}{ F } \\
\cline { 2 - 9 } & \multicolumn{2}{c}{ Female $(\mathrm{n}=20)$} & \multicolumn{2}{c}{ Male $(\mathrm{n}=15)$} & \multicolumn{2}{c}{ Female $(\mathrm{n}=23)$} & \multicolumn{2}{c}{ Male $(\mathrm{n}=7)$} \\
\cline { 2 - 9 } Parameter & Mean \pm SD & Range & Mean \pm SD & Range & Mean \pm SD & Range & Mean \pm SD & Range \\
\hline Age (years) & $69 \pm 11$ & 48 & $67 \pm 10$ & 35 & $81 \pm 6$ & 31 & $78 \pm 6$ & 14 \\
BV/TV (\%) & $12.883 \pm 5.047$ & 19.211 & $17.002 \pm 8.257$ & 26.665 & $13.094 \pm 6.057$ & 22.117 & $13.100 \pm 5.537$ & 16.265 \\
BS/BV (mm ${ }^{-1}$ ) & $13.539 \pm 9.386$ & 25.527 & $9.713 \pm 8.121$ & 20.034 & $11.768 \pm 8.872$ & 22.437 & $12.959 \pm 9.138$ & 22.732 \\
SMI (none) & $1.838 \pm 0.422$ & 1.711 & $1.624 \pm 0.528$ & 1.594 & $1.736 \pm 0.424$ & 1.742 & $1.777 \pm 0.247$ & 0.808 \\
Tb.Th (mm) & $2.515 \pm 3.696$ & 9.506 & $2.844 \pm 3.381$ & 8.903 & $2.608 \pm 3.351$ & 8.199 & $1.951 \pm 2.935$ & 6.327 \\
Tb.N (mm $\left.{ }^{-1}\right)$ & $0.398 \pm 0.290$ & 0.841 & $0.441 \pm 0.384$ & 0.970 & $0.365 \pm 0.307$ & 0.995 & $0.410 \pm 0.279$ & 0.707 \\
Tb.Sp (mm) & $8.378 \pm 12.082$ & 31.458 & $10.621 \pm 12.869$ & 31.142 & $9.381 \pm 12.185$ & 30.879 & $7.295 \pm 11.166$ & 23.007 \\
DA (none) & $0.898 \pm 0.147$ & 0.542 & $0.869 \pm 0.199$ & 0.685 & $0.870 \pm 0.164$ & 0.734 & $0.896 \pm 0.108$ & 0.315 \\
FD (none) & $2.094 \pm 0.113$ & 0.385 & $2.134 \pm 0.121$ & 0.414 & $2.123 \pm 0.081$ & 0.307 & $2.092 \pm 0.079$ & 0.240 \\
\hline
\end{tabular}

Table 4. Spearman correlation coefficients between trabecular microarchitectural parameters and age for coxarthrosis (CA) and fragility fracture $(\mathrm{F})$ groups.

\begin{tabular}{ccccccccc}
\hline & BV/TV (\%) & BS/BV $\left(\mathrm{mm}^{-1}\right)$ & SMI (none) & Tb.Th $(\mathrm{mm})$ & Tb.N $\left(\mathrm{mm}^{-1}\right)$ & Tb.Sp (mm) & DA (none) & FD (none) \\
\hline $\mathrm{CA}$ & -0.213 & -0.030 & 0.311 & 0.057 & -0.166 & 0.172 & -0.113 & -0.268 \\
$\mathrm{~F}$ & -0.305 & 0.034 & 0.136 & -0.058 & -0.182 & 0.133 & 0.104 & -0.209 \\
\hline
\end{tabular}

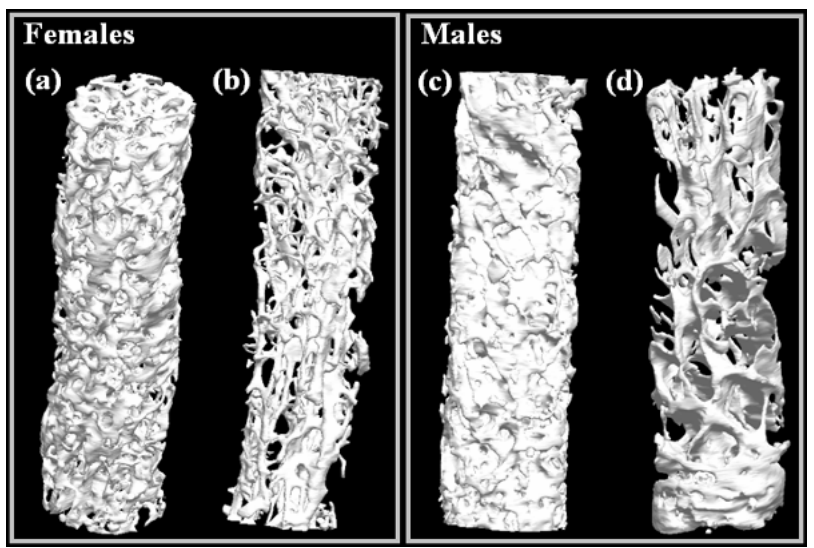

Figure 4. Three-dimensional reconstruction of coxarthrosis samples from a female with 59 years (a) and 75 years (b), and from a male with 60 years (c) and 74 years (d).

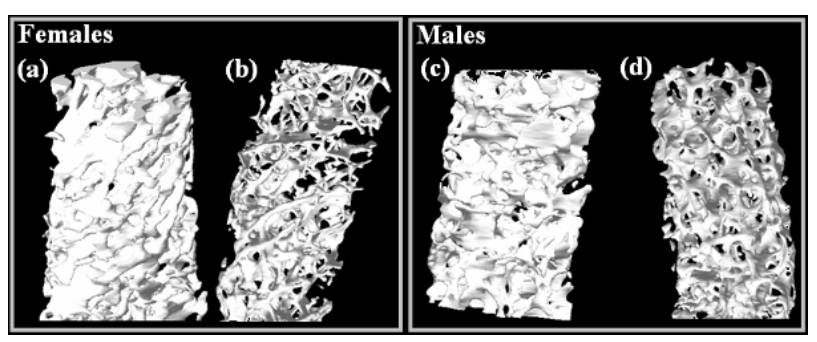

Figure 5. Three-dimensional reconstruction of fragility fracture samples from a female with 76 years (a) and 84 years (b), and from a male with 70 years (c) and 84 years (d).

man coefficient signs, meaning that there are differences between the coxarthrosis specimens and fragility fracture groups. While $\mathrm{BS} / \mathrm{BV}$ and $\mathrm{DA}$ decrease with age on the
CA group, an increase with age was found in the F group. The trabecular thickness has a tendency to increase with age on the coxarthrosis group, while it decreases in fragility fracture group.

Additionally, to evaluate disease-related effects on the relationship between each microarchitectural parameter, the Spearman coefficients were determined for each disease group, and the results are presented in Tables 5 and 6. For the majority of the results, the Spearman statistics revealed similar trends for both disease groups. In general, the microarchitectural parameters are well correlated with BV/TV, showing high values of the coefficients. The worst correlation values were obtained for DA. Trabecular network complexity (FD) and trabecular Structure Model Index are strongly correlated with $\mathrm{BV} / \mathrm{TV}$ and, consequently, with bone relative density.

\section{DISCUSSION}

A better understanding of the trabecular bone microstructure might be relevant for the evaluation of the fracture risk of hip femoral bone. Several factors may affect the structure of bone, as gender, age and disease. The most important age-related change in trabecular structure is bone loss leading to an enhanced risk of fracture. In our work, micro-CT evaluation revealed no significant differences in microarchitectural parameters between diseases groups and gender.

No significant differences were found from the Spearman coefficient determination for age effect, but both diseases presented similar microstructural relationship with age for the same parameters. This is the case of 
Table 5. Spearman correlation coefficients between each trabecular microarchitectural parameter for coxarthrosis (CA) group.

\begin{tabular}{cccccccc}
\hline & BS/BV & SMI & Tb.Th & Tb.N & Tb.Sp & DA & FD \\
\hline BV/TV (\%) & $-0.382^{*}$ & $-0.848^{* *}$ & $0.356^{*}$ & $0.577^{*}$ & $-0.460^{*}$ & 0.064 & $0.943^{* *}$ \\
BS/BV $\left(\mathrm{mm}^{-1}\right)$ & & 0.253 & $-0.989^{* *}$ & $0.453^{*}$ & $-0.539^{*}$ & 0.061 & -0.307 \\
SMI (none) & & & -0.186 & $-0.580^{*}$ & $0.445^{*}$ & -0.195 & $-0.863^{* *}$ \\
Tb.Th (mm) & & & & $-0.479^{*}$ & $-0.555^{*}$ & -0.103 & 0.277 \\
Tb.N (mm (mm $^{-1}$ ( & & & & & $-0.949^{* *}$ & 0.097 & $0.630^{* *}$ \\
Tb.Sp (mm) & & & & & & -0.141 & $-0.501^{*}$ \\
DA (none) & & & & & & & -0.013 \\
\hline
\end{tabular}

${ }^{*} \mathrm{p}<0.05 ;{ }^{* *} \mathrm{p}<0.0001$.

Table 6. Spearman correlation coefficients between each trabecular microarchitectural parameter for fragility fracture (F) group.

\begin{tabular}{|c|c|c|c|c|c|c|c|}
\hline & $\mathrm{BS} / \mathrm{BV}$ & SMI & Tb.Th & Tb.N & Tb.Sp & $\mathrm{DA}$ & FD \\
\hline $\mathrm{BV} / \mathrm{TV}(\%)$ & $-0.515^{*}$ & $-0.804^{* *}$ & $0.500^{*}$ & $0.511^{*}$ & $-0.480^{*}$ & -0.129 & $0.890^{* *}$ \\
\hline $\mathrm{BS} / \mathrm{BV}\left(\mathrm{mm}^{-1}\right)$ & & $0.384^{*}$ & $-0.980^{* *}$ & $0.415^{*}$ & $-0.447^{*}$ & 0.085 & $-0.398^{*}$ \\
\hline SMI (none) & & & -0.334 & $-0.499^{*}$ & $0.463^{*}$ & -0.145 & $-0.848^{* *}$ \\
\hline Tb.Th (mm) & & & & $-0.430^{*}$ & $0.453^{*}$ & -0.150 & $0.389^{*}$ \\
\hline Tb.N $\left(\mathrm{mm}^{-1}\right)$ & & & & & $-0.975^{* *}$ & 0.012 & $0.544^{*}$ \\
\hline Tb.Sp (mm) & & & & & & 0.046 & $-0.489^{*}$ \\
\hline DA (none) & & & & & & & -0.144 \\
\hline
\end{tabular}

BV/TV, Tb.N, FD, SMI and Tb.Sp. In healthy human trabecular bone, BV/TV, Tb.Th and Tb.N, decrease, while Tb.Sp and SMI increased with age [21]. With the exception of $\mathrm{Tb}$.Th, our findings are in accordance with previous studies $[20,21,50]$.

However, the parameters BS/BV, Tb.Th and DA show different Spearman coefficient signs, for the two disease groups, meaning that aging has different effects on some microarchitectural parameters. This indicates different aging mechanisms for different diseases. With aging, trabecular thickness increases in the CA group, but in the F group, it decreases. The increase in trabecular thickness can be attributed to a compensatory mechanism that tries to maintain bone strength, even during bone loss [20].

An increase in the trabecular separation with age can be achieved by an increase in the intertrabecular distance or by the appearance of large areas with no trabeculae [20]. This potentially happens in both CA and F groups.

Structure Model Index indicates the relative proportion of rods and plates in a 3D structure such as trabecular bone. An ideal plate and an ideal cylinder have SMI values of 0 and 3 respectively. Our SMI mean values revealed that both groups present a mixture of plate- and rod-like model microstructure. It is reported [21] that, human healthy trabecular bone structure changes from a plate-like to a more rod-like structure with age. The increase of SMI with age is demonstrated on Table 4, for our two disease groups.
The Degree of Anisotropy mean values are very close to 1 affirming a rather homogeneous trabecular structure in both groups. With aging, DA tends to decrease in the CA group, while it tends to increase in the F group. It is mentioned that in healthy bone, trabeculae with aging seem to align to the direction of principal loading, which means a decrease on DA. Probably the patients with CA have a tendency to follow this mechanism, while the F patients will maintain the bone anisotropy.

The fractal dimension, FD, which is an indicator of surface complexity of an object that quantifies how the object's surface fills the space, tends to decrease with age, which is explained by a decrease in the bone volume fraction.

The correlation between microarchitectural parameters, determined by the Spearman statistics, shows that the majority of the parameters are well correlated with BV/TV. Moreover, our results are consistent with previous studies for which $\mathrm{Tb}$.Th and Tb.N presented positive significant coefficients with BV/TV, while Tb.Sp showed negative significant coefficients [35]. These findings confirm the very important correlation between BV/TV and trabecular morphometric parameters (Tb.N, Tb.Th and Tb.Sp) that influence porosity and, consequently trabecular bone density.

\section{CONCLUSIONS}

We emphasize the need for a preliminary study on mi- 
cro-CT variables prior to extending it to a large number of samples. In fact, comparison with literature data is not straightforward, even if the same device is used. Changes in the parameters may bias the reconstructed images, giving rise to a high variability of the $3 \mathrm{D}$ microarchitectural parameters.

As on other works, bone volume fraction BV/TV mean values and its good correlation with the other microarchitectural parameters demonstrated the great importance of this parameter in trabecular structural prediction.

Gender, age and disease variations showed no signifycant effects on the microarchitectural parameters of trabecular bone.

As for healthy bone, there seems to be effects of aging in the microarchitecture of bone. Age-related changes in the microstructure of trabecular bone are not the same in different pathologies, such as, coxarthrosis and fragility fracture. With aging, bone specific surface, and the degree of anisotropy decrease in the CA and increase in the $\mathrm{F}$ group, while trabecular thickness increases in the CA group and decreases in the $\mathrm{F}$ group. This means that, depending on the disease, the age effects in the microbarchitecture of bone are different.

\section{ACKNOWLEDGEMENTS}

AC Vale would like to acknowledge the Portuguese research foundation FCT (Fundação para a Ciência e Tecnologia) for providing financial support (SFRH/BD/48100/2008). MFC Pereira and A Maurício acknowledge FEDER Funds through Programa Operacional Factores de Competitividade - COMPETE, and FCT Project PEst-OE/CTE/UI0098/2011.

\section{REFERENCES}

[1] Feldkamp, L.A., Goldstein, S.A., Parfitt, A.M., et al. (1989) The direct examination of three-dimensional bone architecture in vitro by computed tomography. Journal of Bone and Mineral Research, 4, 3-11. doi:10.1002/jbmr.5650040103

[2] Kim, D.G., Christopherson, G.T., Dong, X.N., et al. (2004) The effect of microcomputed tomography scanning and reconstruction voxel size on the accuracy of stereological measurements in human cancellous bone. Bone, 34, 13751382. doi:10.1016/j.bone.2004.09.007

[3] Bouxsein, M.L., Boyd, S.K., Christiansen, B.A., et al. (2010) Guidelines for assessment of bone microstructure in Rodents using micro-computed tomography. Journal of Bone and Mineral Research, 25, 1468-1486. doi:10.1002/jbmr.141

[4] Griffith, J.F. and Genant, H.K. (2008) Bone mass and architecture determination: State of the art. Best Practice \& Research Clinical Endocrinology \& Metabolism, 22, 737-764. doi:10.1016/i.beem.2008.07.003

[5] Chappard, D., Baslé, M.-L., Legrand, E. and Audran, M. (2008) Trabecular bone microarchitecture: A review. Morphologie, 92, 162-170. doi:10.1016/j.morpho.2008.10.003
[6] Benhamou, C.-L. and Roux, C. (2007) First meeting on bone quality, abbaye des Vaux de Cernay, France, 15-16 June 2006: Bone architecture. Osteoporosis International, 18, 837-889. doi:10.1007/s00198-007-0366-4

[7] Lespessailles, E., Chappard, C., Bonnet, N. and Benhamou, C.L. (2006) Imaging techniques for evaluating bone microarchitecture. Joint Bone Spine, 73, 254-261. doi:10.1016/i.jbspin.2005.12.002

[8] Adams, J.E. (2009) Quantitative computed tomography. European Journal of Radiology, 71, 415-424. doi:10.1016/j.ejrad.2009.04.074

[9] Steines, D., Liew, S.W., Arnaud, C., Vargas-Voracek, R., Nazarian, A., Müller, R., Snyder, B., Hess, P. and Lang, P. (2009) Radiographic trabecular 2D and 3D parameters of proximal femoral bone cores correlate with each other and with yield stress. Osteoporos International, 20, 1929 1938. doi:10.1007/s00198-009-0908-Z

[10] Ding, M. and Hvid, I. (2000) Quantification of age-related changes in the structure model type and trabecular thickness of human tibial cancellous bone. Bone, 26, 291295. doi:10.1016/S8756-3282(99)00281-1

[11] Rüegsegger, P., Koller, B. and Müller, R. (1996) A microtomographic system for the nondestructive evaluation of bone architecture. Calcified Tissue International, 58, 24-29. doi:10.1007/BF02509542

[12] Ciarelli, M.J., Goldstein, S.A., Kuhn, J.L., Cody, D.D. and Brown, M.B. (1991) Evaluation of orthogonal mechanical properties and density of human trabecular bone from the major metaphyseal regions with materials testing and computed tomography. Journal of Orthopaedic Research, 9, 674-682. doi:10.1002/jor.1100090507

[13] Müller, R. and Rüegsegger, P. (1997) Micro-tomographic imaging for the nondestructive evaluation of trabecular bone architecture. Studies in Health Technology and Informatics, 40, 61-79.

[14] Arlot, M., Burt-Pichat, B., Roux, J., et al. (2008) Microarchitecture influences microdamage accumulation in human vertebral trabecular bone. Journal of Bone and Mineral Research, 23, 1613-1618. doi:10.1359/jbmr.080517

[15] Hildebrand, T., Laib, A., Müller, R., Dequeker, J. and Rüegsegger, P. (1999) Direct threedimensional morphometric analysis of human cancellous bone: Microstructural data from spine, femur, iliac crest, and calcaneus. Journal of Bone and Mineral Research, 14, 1167-1174. doi:10.1359/jbmr.1999.14.7.1167

[16] Tamminen, I.S., Isaksson, H., Aula, A.S., et al. (2011) Reproducibility and agreement of micro-CT and histomorphometry in human trabecular bone with different metabolic status. Journal of Bone and Mineral Metabolism, 29, 442-448. doi:10.1007/s00774-010-0236-6

[17] Müller, R., Van Campenhout, H., Van Damme, B., et al. (1998) Morphometric analysis of human bone biopsies: A quantitative structural comparison of histological sections and micro-computed tomography. Bone, 23, 59-66. doi:10.1016/S8756-3282(98)00068-4

[18] Djuric, M., Djonic, D., Milovanovic, P., et al. (2010) Region-specific sex-dependent pattern of age-related changes of proximal femoral cancellous bone and its im- 
plications on differential bone fragility. Calcified Tissue International, 86, 192-201.

doi:10.1007/s00223-009-9325-8

[19] Macho, G.A., Abel, R.L. and Schutkowski, H. (2005) Age changes in bone microstructure: Do they occur uniformly? International Journal of Osteoarchaeology, 15, 421-430. doi:10.1002/oa.797

[20] Cui, W.-Q., Won, Y.-Y., Baek, M.-H., et al. (2008) Age- and region-dependent changes in three-dimensional microstructural properties of proximal femoral trabeculae. Osteoporosis International, 19, 1579-1587. doi:10.1007/s00198-008-0601-7

[21] Chen, H., Zhou, X., Shoumura, S., Emura, S. and Bunai, Y. (2010) Age- and gender-dependent changes in three-dimensional microstructure of cortical and trabecular bone at the human femoral neck. Osteoporosis International, 21, 627-636. doi:10.1007/s00198-009-0993-z

[22] Issever, A.S., Vieth, V., Lotter, A., et al. (2002) Local differences in the trabecular bone structure of the proximal femur depicted with high-spatial-resolution MR imaging and multisection CT. Academic Radiology, 9, 13951406. doi:10.1016/S1076-6332(03)80667-0

[23] Nazarian, A., Müller, J., Zurakowski, D., Müller, R. and Snyder, B.D. (2007) Densitometric, morphometric and mechanical distributions in the human proximal femur. Journal of Biomechanics, 40, 2573-2579. doi:10.1016/j.jbiomech.2006.11.022

[24] Bauer, J.S. and Link, T.M. (2009) Advances in osteoporosis imaging. European Journal of Radiology, 71, 440449. doi:10.1016/j.ejrad.2008.04.064

[25] Campbell, G.M., Buie, H.R. and Boyd, S.K. (2008) Signs of irreversible architectural changes occur early in the development of experimental osteoporosis as assessed by in vivo micro-CT. Osteoporosis International, 19, 14091419. doi:10.1007/s00198-008-0581-7

[26] Laib, A., Barou, O., Vico, L., et al. (2000) 3D microcomputed tomography of trabecular and cortical bone architecture with application to a rat model of immobilisation osteoporosis. Medical and Biological Engineering and Computing, 38, 326-332. doi:10.1007/BF02347054

[27] Lill, C.A., Gerlach, U.V., Eckhardt, C., et al. (2002) Bone changes due to glucocorticoid application in an ovariectomized animal model for fracture treatment in osteoporosis. Osteoporosis International, 13, 407-414. doi:10.1007/s001980200047

[28] Xiang, A., Kanematsu, M., Kumar, S., et al. (2007) Changes in micro-CT $3 \mathrm{D}$ bone parameters reflect effects of a potent cathepsin $\mathrm{K}$ inhibitor (SB-553484) on bone resorption and cortical bone formation in ovariectomized mice. Bone, 40, 1231-1237. doi:10.1016/j.bone.2007.01.010

[29] Nazarian, A., Snyder, B.D., Zurakowski, D. and Müller, R. (2008) Quantitative micro-computed tomography: A non-invasive method to assess equivalent bone mineral density. Bone, 43, 302-311. doi:10.1016/j.bone.2008.04.009

[30] Sun, S.-S., Ma, H.-L., Liu, C.-L., et al. (2008) Difference in femoral head and neck material properties between osteoarthritis and osteoporosis. Clinical Biomechanics, 23, 39-47. doi:10.1016/j.clinbiomech.2007.11.018
[31] Genant, H.K., Engelke, K. and Prevrhal, S. (2008) Advanced CT bone imaging in osteoporosis. Rheumatology, 47, 9-16. doi:10.1093/rheumatology/ken180

[32] Wu, Z.-X., Lei, W., Hu, Y.Y., et al. (2008) Effect of ovariectomy on $\mathrm{BMD}$, micro-architecture and biomechanics of cortical and cancellous bones in a sheep model. Medical Engineering \& Physics, 30, 1112-1118. doi:10.1016/j.medengphy.2008.01.007

[33] Campbell, G.M., Ominsky, M.S. and Boyd, S.K. (2011) Bone quality is partially recovered after the discontinuation of RANKL administration in rats by increased bone mass on existing trabeculae: An in vivo micro-CT study. Osteoporosis International, 22, 931-942. doi:10.1007/s00198-010-1283-5

[34] Kapadia, R.D., Stroup, G.B., Badger, A.M., et al. (1998) Applications of micro-CT and MR microscopy to study pre-clinical models of osteoporosis and osteoarthritis. Technology and Health Care, 6, 361-372.

[35] Zhang, Z.M., Li, Z.C., Jiang, L.S., Jiang, S.D. and Dai, L.Y. (2010) Micro-CT and mechanical evaluation of subchondral trabecular bone structure between postmenopausal women with osteoarthritis and osteoporosis. Osteoporosis International, 21, 1383-1390. doi:10.1007/s00198-009-1071-2

[36] Ito, M., Nakamura, T., Matsumoto, T., et al. (1998) Analysis of trabecular microarchitecture of human iliac bone using microcomputed tomography in patients with hip arthrosis with or without vertebral fracture. Bone, 23, 163-169. doi:10.1016/S8756-3282(98)00083-0

[37] Chappard, C., Peyrin, F., Bonnassie, A., et al. (2006) Subchondral bone micro-architectural alterations in osteoarthritis: A synchrotron micro-computed tomography study. Osteoarthritis Cartilage, 14, 215-223. doi:10.1016/j.joca.2005.09.008

[38] Tassani, S., Particelli, F., Perilli, E., et al. (2011) Dependence of trabecular structure on bone quantity: A comparison between osteoarthritic and non-pathological bone. Clinical Biomechanics, 26, 632-639. doi:10.1016/j.clinbiomech.2011.01.010

[39] Nazarian, A. and Müller, R. (2004) Time-lapsed microstructural imaging of bone failure behaviour. Journal of Biomechanics, 37, 55-65. doi:10.1016/S0021-9290(03)00254-9

[40] Nazarian, A., Hermannsson, B.J., Müller, J., Zurakowski, D. and Snyder, B.D. (2009) Effects of tissue preservation on murine bone mechanical properties. Journal of Biomechanics, 42, 82-86. doi:10.1016/j.jbiomech.2008.09.037

[41] Nazarian, A., Entezari, V., Vartanians, V., Müller, R. and Snyder, B.D. (2009) An improved method to assess torsional properties of rodent long bones. Journal of Biomechanics, 42, 1720-1725. doi:10.1016/j.jbiomech.2009.04.019

[42] Nazarian, A., Bauernschmitt, M., Eberle, C., Meier, D., Müller, R. and Snyder, B.D. (2008) Design and validation of a testing system to assess torsional cancellous bone failure in conjunction with time-lapsed micro-computed tomographic imaging. Journal of Biomechanics, 41, 34963501. doi:10.1016/j.jbiomech.2008.09.014 
[43] Verdelis, K., Lukashova, L., Atti, E. et al. (2011) MicroCT morphometry analysis of mouse cancellous bone: Intra- and inter-system reproducibility. Bone, 49, 580-587. doi:10.1016/j.bone.2011.05.013

[44] Beaupied, H., Chappard, C., Basillais, A., et al. (2006) Effect of specimen conditioning on the microstructural parameters of trabecular bone assessed by micro-computed tomography. Physics in Medicine and Biology, 51, 4621-4634. doi:10.1088/0031-9155/51/18/011

[45] Dezna, C. and Sheehan, B. (1973) Theory and practice of histotechnology. 1st Edition, C.V. Mosby Company, Saint Louis.

[46] Erben, R.G. (1997) Embedding of bone samples in methylmethacrylate: an improved method suitable for bone histomorphometry, histochemistry, and immunohistochemistry. Journal of Histochemistry \& Cytochemistry, 45,
307-313. doi:10.1177/002215549704500215

[47] Odgaard, A. (1997) Three-dimensional methods for quantification of cancellous bone architecture. Bone, 20, 315328. doi:10.1016/S8756-3282(97)00007-0

[48] Parfitt, A., Drezner, M., Glorieux, F. et al. (1987) Bone histomorphometry: Standardization of nomenclature, symbols, and units. Journal of Bone and Mineral Research, 2, 595-610. doi:10.1002/jbmr.5650020617

[49] SkyScan 1172, N.V. Vluchtenburgstraat 3C 2630-Kontick Belgium. http://www.skyscan.be/home.htm

[50] Viguet-Carrin, S., Follet, H., Gineyts, E., et al. (2010) Association between collagen cross-links and trabecular microarchitecture properties of human vertebral bone. Bone, 46, 342-347. doi:10.1016/j.bone.2009.10.001 